\title{
VLSI Architecture for Optimization Transform Technique based on Compression of ECG Signals
}

\author{
Ashraf Mohamed Ali \\ Department of Electronics and \\ Communications \\ Sinai University, Egypt
}

\author{
Waleed Elnahel \\ Department of Electronics and \\ Communications \\ October University for Modern \\ Sciences and Arts, Egypt
}

\author{
Hatem M. Zakaria \\ Electrical Engineering Department, \\ Benha Faculty of Engineering, \\ Benha University, Egypt
}

\begin{abstract}
The measurement of electrical activity of the heart via electrodes is named as Electrocardiography (ECG). An efficient compression technique using the compressive sensing method is required. Compressive Sensing (CS) holds the promise to be a key for acquisition and reconstruction of sparse signals. The reconstruction of such signals makes sampling rates below Nyquist rate. In this work, a novel framework was proposed that is based on the idea of CS theory for the compression of mother and fetal heart beats. The proposed scheme is based on the sparse representation of the components derived from the curvelet transform of the original Electrocardiogram (ECG) signal. The ECG signals may be approximated by a few coefficients that can be taken from a wavelet basis. This fact allows a compressed sensing approach for ECG signal compression to be introduced and to be a domain of search. ECG signals illustrate redundancy between adjacent heart beats. This redundancy implies a high fraction of common support between consecutive heart beats. The main contribution of this paper lies in the using of curvelet transform in order to generate sparsity in ECG signal. This transformation is considered an excellent approach as illustrated in this paper. Simulation results represent a better approach than Discrete Wavelet Transform (DWT) that is based on compression of ECG. MIT-BIH database is used for experimentation. The MIT-BIH database contains different kinds of ECG signals that include both abnormal ECG and normal ECG, which have different sampling rates. MATLAB tool is used for simulation purpose. The novelty of the method is that the Compression Ratio (CR) achieved by detail coefficients is better. The performance measure of the reconstructed signal is carried out by Percentage Root Mean Difference (PRD). This paper also introduces the efficient realization of the different transformation techniques using FPGA. Thus the contribution of this paper lies into two main parts. The first part is specialized in determining the proper transformation that is used in the compression of ECG signals. The second part of the contribution is summarized in using suitable hardware to implement this design. Architecture can be based on the ideas of parallelism and pipelining to get the minimum throughput and speed. Architecture is cascade and simple for calculating curvelet coefficients. The reduction of the memory size can be done by splitting ROM table. The description and functionalities of the design are modeled by Verilog HDL. The simulation and synthesis methodology are used on Virtex-II Pro FPGA that uses less number of resources of the FPGA.
\end{abstract}

\section{General Terms}

Discrete Wavelet Transform; Performance Measure; Compression

\author{
Keywords \\ Compressive sensing; Sparse; Sampling rates; Heart beats; \\ Compression ratio; FPGA
}

\section{INTRODUCTION}

The traditional approach which is used for recovery of signals from measured data follows the theorem of Shannon in sampling. This theorem states that the sampling rate must be at least twice the highest frequency in order to reconstruct the signal. In the same way, linear algebra has the fundamental theorem. This theorem suggests that the number of generating samples (measurements) of a discrete finite-dimensional signal should have in the minimum case the same length of the signal to help in the recovery process [1]. The theory of CS suggests that the randomized low sampling rate provides an efficient method for high sampling rate of exploiting the prior of sparsity and this in the case of recovering the original signals[2],[3]. CS method is used to transform the signal into low dimensional measurement domain with under-sampling and it is also known in the recent years as compressive sampling [4], [5]. In this paper, the main target is to use a CS technique to enhance the compression of ECG signals. This is considered an important application in biomedical engineering because heart disease leads to the mortality of most people in the world [6]. The modern society is today threatened by an incipient health care delivery crisis caused by the current demographic and lifestyle trends [7]. The ECG provides information about the heart. The ECG is a biological signal which generally changes its physiological and statistical property with respect to time. This signal can be considered as a diagnostic tool which graphically measures and records the electrical signal of heart beats. This measurement is in details and can be used in the extraction of the main features of ECG signal [8]. In studying such types of signals, wavelet transforms can be used. The most striking waveform when taking in consideration the ECG is QRS wave complex, which gives the R wave peak [9]. Wavelet Transform (WT) can be considered as a time-frequency signal analysis tool and it is used in a wide variety of applications that include signal and image coding. The fast implementation of WT can be carried out using Sub-Band (SB) filter banks [10]. The main question is, for a sparse signals that is represented in the time domain, how can anyone obtain a recursive solution that enhances the accuracy of simple CS by using past observations? Recursive means a solution that uses only the previous signal estimation and the currentobservation vector at the current time [11]. In the recent study, Candes et al. presented a new member that can be within the family of wavelet transform. This member is called the curvelet transform. The first generation curvelet transform is based on the concepts of ridgelet transform. The main target of this transformation is to face the limitations of traditional multi-scale representations of the signals. [12]. The 
important factor to be considered in compression of ECG signal is to obtain the maximum data reduction. That is to solve the defects of wavelets in the higher order of dimensions. Cand'es and Donoho [13], [14] recently explained a new system of representations. This system is named ridgelets and it can be represented effectively as line singularities in 2-D. The main target of this transformation is to use the Radon transform as a way to transform a line singularity into a point singularity [15]. The ECG signal is shown in figure 1 . This signal consists of $\mathrm{P}, \mathrm{Q}, \mathrm{R}, \mathrm{S}, \mathrm{T}$ and $\mathrm{U}$ waves. The name of the first positive wave is the $P$ wave. The QRS complex wave consists of $\mathrm{Q}, \mathrm{R}$ and $\mathrm{S}$ waves. The $\mathrm{T}$ wave is produced by ventricular re-polarization and its shape is smooth. The $\mathrm{U}$ wave comes after the $\mathrm{T}$ wave and before the $\mathrm{P}$ wave of the next cycle. In the present study, an algorithm is developed in a way to extract the ECG features automatically. The developed algorithm begins by decomposing the original ECG signal by using DWT and Daubahies Wavelet (db6) as mother wavelet. It begins by removing the low frequency components that contain the Baseline Drift De-trending and the removing of high frequency components that contain the noise component. After that, it uses the preprocessed signal to help in extraction of features from the ECG signal automatically [16]. The ECG signals in the recent study are collected from MIT/BIH database using Physionet database and it can be stored in a text format [17]. This paper is structured as follows: section 2 explains the related work, including the two most recent techniques in this domain of search, section 3 demonstrates trends and challenges for obtaining a sparse signal of ECG, section 4 explains the proposed technique and its analysis, section 5 describes the performance measure to validate the technique used in this research, section 6 demonstrates VLSI architecture, section 7 covers the MATLAB simulation results with discussions, section 8 presents FPGA realization, section 9 introduces the conclusions obtained from this work.

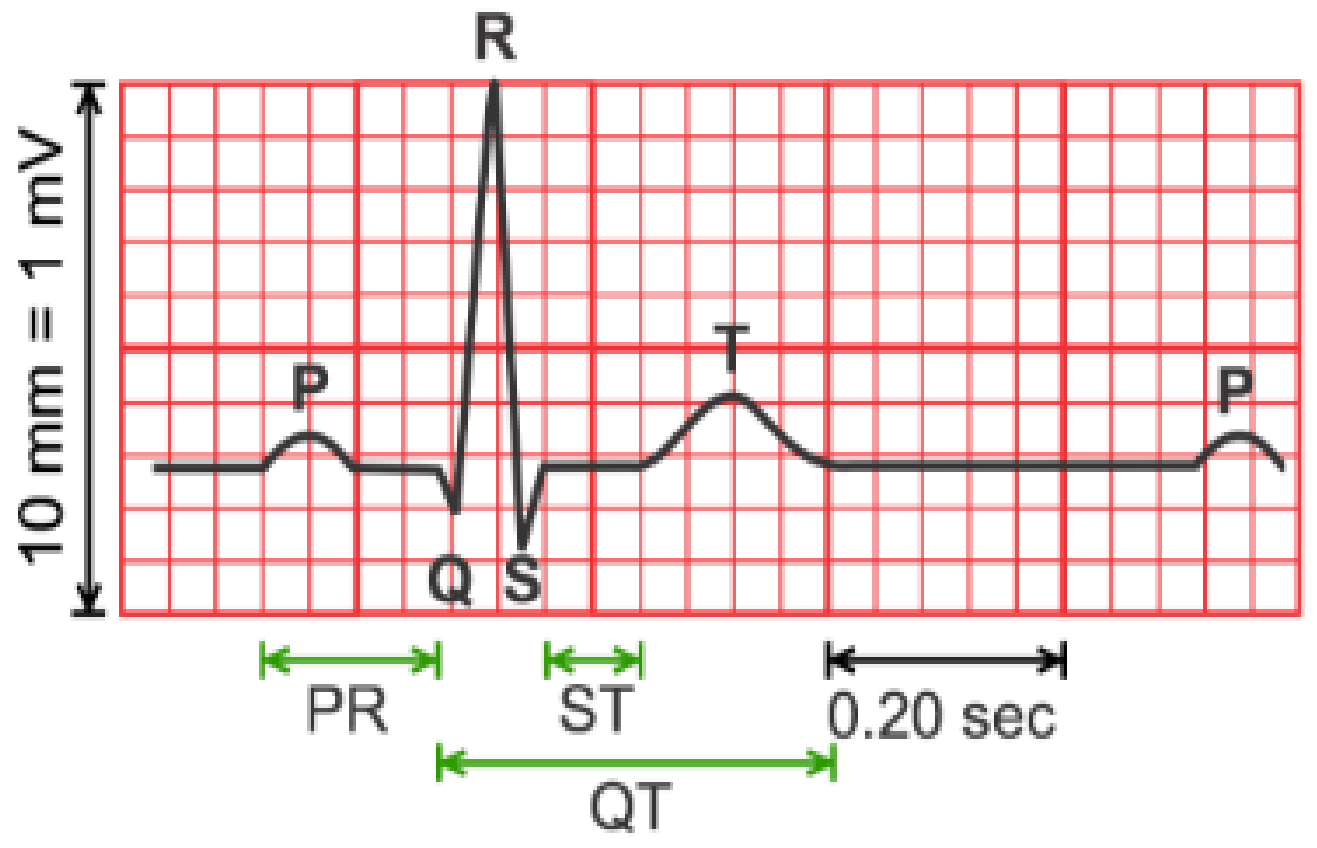

Fig.1 Typical ECG signal [6].

\section{RELATED WORK}

The incorporation of a sliding window whose fixed length can be determined by the sparsity of the heart beats can be presented as related work. The length of the window should be short and this is due to the generation of an approximate real time transmission, At the same time, we want to incorporate enough number of heart beats on the window to can reconstruct the signal with fewer number of samples [18], [19]. Another approach proposes a wavelet ECG that is based on the Set Partitioning In Hierarchical Trees (SPIHT) compression algorithm. The SPIHT algorithm has achieved a great success in the field of image coding. The authors modified the algorithm to be used in the one-dimensional case and this helps them to apply it to compression of ECG data [20], [21].

\section{TRENDS AND CHALLANGES}

Selection of suitable transformations to obtain sparse signal of ECG can be considered as the most challenge problems. This sparse signal is useful to help in applying CS technique and to guarantee the reconstruction of the original signal. The main difference between each transformation and another is their ability in the reconstruction of the sparse signal of ECG. The suitable transformation to be used in this area of search is determined by their excellent performance in reconstruction of the original signal. The most transformation techniques in this domain of search are Wavelet Transform [22], [23], Discrete Cosine Transform [24] and Walsh Hadamrd Transform [25], [26]. Here in this work the introduced transformation is curvelet transform that gives excellent results. The following sections will explain this technique and will show how it presents an excellent performance. Through analysis and results, we conclude that this transformation is the prefect transformation in obtaining the sparse signal of ECG.

\section{THE PROPOSED TECHNIQUE}

Here in this section, the proposed technique is introduced and explained. This section also makes analysis of the proposed approach to show how this approach obtains the sparse signal of ECG in an efficient way. 


\subsection{Materials and Methods}

The proposed algorithm that can be used for ECG signal compression and recovery is illustrated in figure 3. As demonstrated in this figure, there are four compressing stages and three recovery stages. For compression, the first stage is to obtain the sparsity of the ECG signal using ridgelet transform. The second stage is to apply the CS technique on the sparse signal. The third stage is to quantize the sampled values of the signal. The final stage is to encode the quantized values. The data transmitted to a reconstruction stage can be considered as the data gets out of the last compression stage. Figure 3 also shows the recovery stage that is the reverse order of the compression stage. The first recovery stage

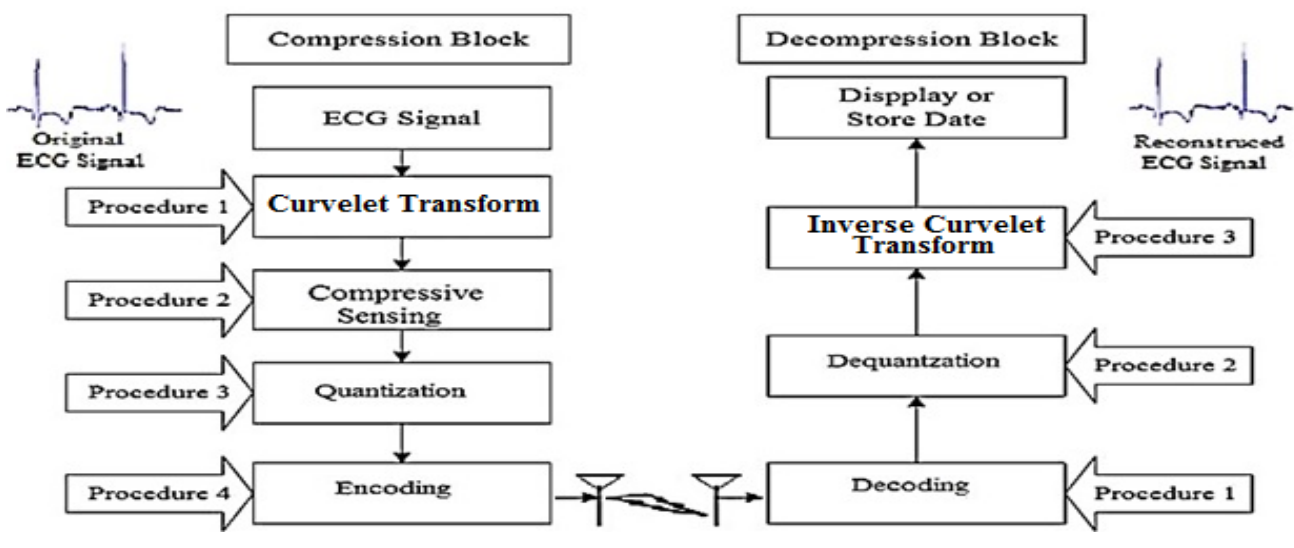

Fig. 3. Block Diagram of the proposed system

\subsection{Analysis of The Proposed Approach}

Curvelet transform is a recent transform, which can be used in a line or super-plane singularities [27], [28]. Considering a function $\mathrm{f}(\mathrm{x})$, its Continuous Ridgelet Transform (CRT) in $\mathrm{R}^{2}$ is represented by the following equation:

$C R T_{f}(a, b, \theta)=\int_{R^{2}} \Psi_{a, b, \theta}\left(X_{1}, X_{2}\right) f\left(X_{1}, X_{2}\right) d X_{1} d X_{2}$

Given that, the ridgelet $\psi_{\mathrm{a}, \mathrm{b}, \theta}$ in 2-D can be defined as a function of a wavelet type function in 1-D $\psi(\mathrm{X})$ in the given equation:

$\psi_{a, b, \theta}\left(X_{1}, X_{2}\right)=a^{-1 / 2} \psi \frac{\left(X_{1} \cos \theta+X_{2} \sin \theta-b\right)}{a}$

The previous equation gives the description of wavelet by an angle $\theta$ and taking in consideration that $X_{1} \cos \theta+X_{2} \sin \theta=C$, given that $\mathrm{C}$ is constant. The Continuous Wavelet Transform (CWT) in $R^{2}$ of $f(X)$ is represented by the given equation

$C W T_{f}\left(a_{1}, a_{2}, b_{1}, b_{2}\right)=\int_{R^{2}} \Psi_{\left(a_{1}, a_{2}, b_{1}, b_{2}\right)}(X) f(X) d X_{1} d X_{2}$

Given that, the wavelets in 2-D are described as multiplication of 1-D wavelets,

$$
\Psi_{\left(a_{1}, a_{2}, b_{1}, b_{2}\right)}(X)=\Psi_{a_{1}, b_{1}}\left(X_{1}\right) \psi_{a_{2}, b_{2}}\left(X_{2}\right)
$$

1-D has the given equation

$\psi_{a, b}\left(X_{1}\right)=a^{-1 / 2} \psi\left(\frac{t-a}{b}\right)$

As noticed from Equations (3) and (5), the CRT is similar to the 2-D CWT, taking in consideration that the point parameters $\left(b_{1}, b 2\right)$ are replaced with the line parameters $(b$, $\theta)$. This transformation is represented as: applies decoding algorithm to the compressed data. The second stage is to dequantize the data to obtain the sparse signal. The third stage is to apply inverse ridgelet transform in the sparse signal, which is considered to be the reconstructed ECG signal. In this way this block diagram summarized the proposed technique. The next section illustrates the effect of introducing ridgelet transform in obtaining the sparse signal which is considered the most contribution of this paper. Hardware design can be done using the FPGA module. The challenge at this point is to make the design performance more efficient in terms of simplicity of the design by using less number of resources.

$R_{f}(\theta, t)=\int_{R^{2}} f(X) \delta\left(X_{1} \cos \theta+X_{1} \sin \theta-t\right) d x_{1} d x_{2}$

Where delta is the function of Dirac delta. As the result, the RT can be considered as a 1-D wavelet transform applied to the segments of the Radon transform

$$
C R T_{f}(a, b, \theta)=a^{-1 / 2} \int_{R} \psi\left(\frac{t-b}{a}\right) R_{f}(\theta, t) d t
$$

Equation (7) is applied to ECG signal in this work to obtain the sparse signal. In this way CS can be applied to the obtained sparse signal

\section{PREFORMANCE MEASURE}

The Compression Ratio (CR), the Percentage Root Mean Difference (PRD), the Peak Signal-to-Noise Ratio (PSNR) and the Mean Square Error (MSE) will be used as a performance measure to validate the proposed approach and to determine its robustness in its area of search. Here in this section, these parameters are discussed briefly.

\subsection{Compression Ratio}

The compression ratio (CR) can be defined as the ratio of the bit rate that represents the original signal to bit rate that is needed to save the compressed signal. All algorithms of compression can be used in minimization of data storage by eliminating the redundancy if it is available. This concept helps in increasing the compression ratio [29].

$$
\begin{aligned}
& \text { Compression Ratio (CR) } \\
& =\frac{\text { Bit Rate Of Original ECG Signal }}{\text { Bit Rate of Compressed ECG Signal }}
\end{aligned}
$$




\subsection{Percentage Root Mean Difference (PRD)}

The PRD gives an indication of the difference between the original ECG data and the recovered data. This concept is utilized for indicating the distortions in recovering biomedical signals such as ECG signals. The PRD has three types for ECG data compression [6], which are shown in equations (9), (10) and (11). Given $x_{1}(n)$ is the original signal of length $N$ and $\mathrm{x}_{2}(\mathrm{n})$ is the recovered signal of length $\mathrm{N}$.

$$
\begin{gathered}
P R D_{0}=\sqrt{\frac{\sum_{n=1}^{N}\left[x_{1}(n)-x_{2}(n)\right]^{2}}{\sum_{n=1}^{N} x_{1}(n)^{2}}} \times 100 \% \\
P R D_{1}=\sqrt{\frac{\sum_{n=1}^{N}\left[x_{1}(n)-x_{2}(n)\right]^{2}}{\sum_{n=1}^{N}\left[x_{1}(n)-\text { offset }\right]^{2}}} \\
P R D_{1}=\sqrt{\frac{\sum_{n=1}^{N}\left[x_{1}(n)-x_{2}(n)\right]^{2}}{\sum_{n=1}^{N}\left[x_{1}(n)-\text { offset }\right]^{2}}}
\end{gathered}
$$

In this work, the second approach which is $\mathrm{PRD}_{1}$ is applied to define PRD. Table 1 demonstrates the quality according to the range of $\mathrm{PRD}_{1}$.

Table 1. Quality according to the range of $\mathrm{PRD}_{1}[6]$

\begin{tabular}{|c|c|}
\hline Range of PRD $_{\mathbf{1}}$ & Quality \\
\hline $0 \% \leq \mathrm{PRD}_{1}<2 \%$ & Very Good \\
\hline $2 \% \leq \mathrm{PRD}_{1}<9 \%$ & Good \\
\hline $9 \% \leq \mathrm{PRD}_{1}<19 \%$ & Not Good \\
\hline $19 \% \leq \mathrm{PRD}_{1}<60 \%$ & Bad \\
\hline
\end{tabular}

\subsection{Mean Square Error (MSE)}

Mean Square Error measures the amount by which the reconstructed signal differs from the original signal [30]. MSE is defined as follows:

$$
\sigma^{2}=\frac{1}{N} \sum_{n=1}^{N}\left(x_{n}-y_{n}\right)^{2}
$$

Where $x_{n}, y_{n}$ and $N$ are the original signal, the reconstructed signal and the length of the signal respectively.

\subsection{Peak Signal to Noise Ratio (PSNR)}

Peak Signal to Noise Ratio has the following definition [31]

$$
P S N R=10 \log _{10} \frac{x_{\text {peak }}^{2}}{\sigma_{d}^{2}}
$$

Where $\mathrm{x}_{\text {peak }}$ is the peak of the input data sequence and $\sigma_{d}^{2}$ is the MSE.

\section{VLSI ARCHITECTURE}

The hardware implementation of ACS is shown in figure 4. The sampled data $Y$ and $\Psi$ are saved in external double-data rate synchronous dynamic random access memory. The processing element is modularized circuit which is for the purpose of calculating the product of complex vectors and it is configured to the default value 64 bit width. The Xilinx Logic CORE IP core is for the multiplying accumulator for two fixed point vectors. FP1 is a floating-to-fixed point conversion. FP2 is for fixed-to-floating point conversion. The output of FP1 and FP2 holds a 32-bit width; 16 for integer part and 16 for fraction part. To process data by FFT IP core, they are converted into 16 bits for fractional part and 16 bits for the integer part. To perform 2048-point transform the output of FFT IP is 44 bit width, 28 for integer part and 16 for the fractional part. The FFT IP core prevents the RAMs from storing intermediate data.

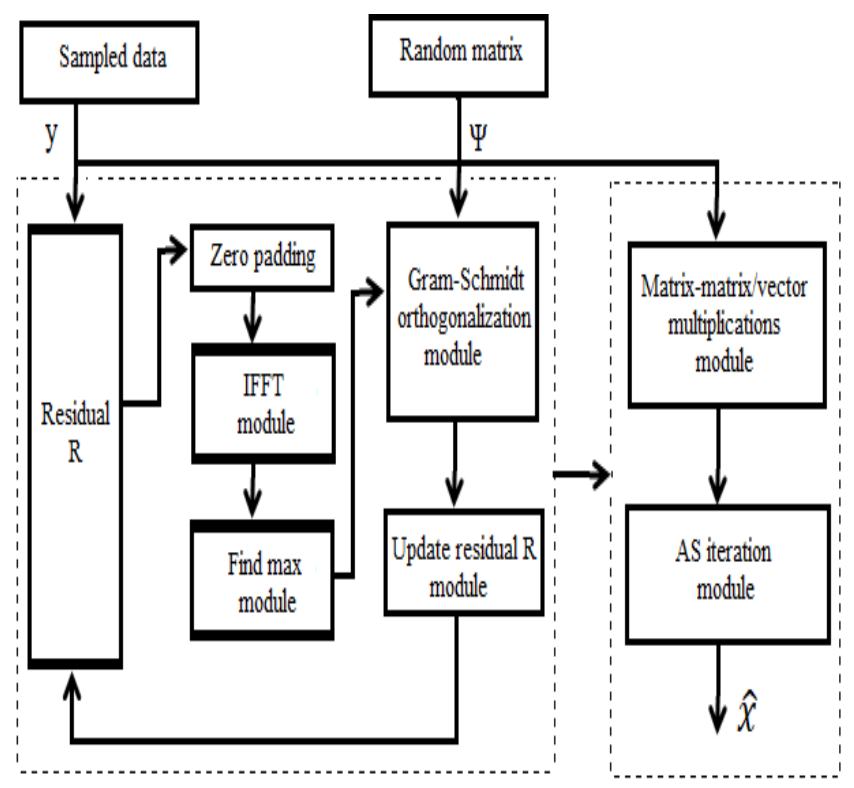

Fig.4. Detailed architecture diagram of top-level design

\section{MATLAB SIMULATION RESULTS}

MIT-BIH database was chosen for testing the signals. The software was done via MATLAB tool. Many researchers have introduced different compression algorithms for ECG signals. In this research, an evaluation of data compression algorithms uses these four parameters that are CR, PRD, RMS and PSNR. Figures 5 and 6 show the original ECG signal and recovered signal for normal persons after using the proposed technique compared with the two most recent techniques in the domain of search for different records. Figures 7 and 8 show the original ECG signal and recovered signal for patient persons. Figures 8 and 9 illustrate the compression ratio versus the number of samples of normal and patient persons respectively for different approaches. Figures 10 and 11 illustrate the PRD versus the number of samples of normal and patient persons respectively for different approaches. To estimate the algorithm performance accurately, the results were compared to the performance of the previous compression algorithms. These comparisons are illustrated in tables 4 and 5 for normal and patient persons respectively to show to what extends the proposed technique achieved an excellent result. The results in these tables are taken for a number of samples equal to 1000. Experimental results indicate that the ridgelet transform introduces an excellent representation for ECG Signals that are smooth away from line discontinuities. By applying a small number of samples that is represented as sparse signal, the ECG can be reconstructed with high accuracy through the proposed optimization stages. CS based ECG compression is accordingly shown to achieve an excellent result relative to its DWT based counterpart for good reconstruction quality. Ridgelet technique is the clear choice for ECG signals compression. This because Ridgelet Transform is localized and has a non-stationary feature of the curvelet. 

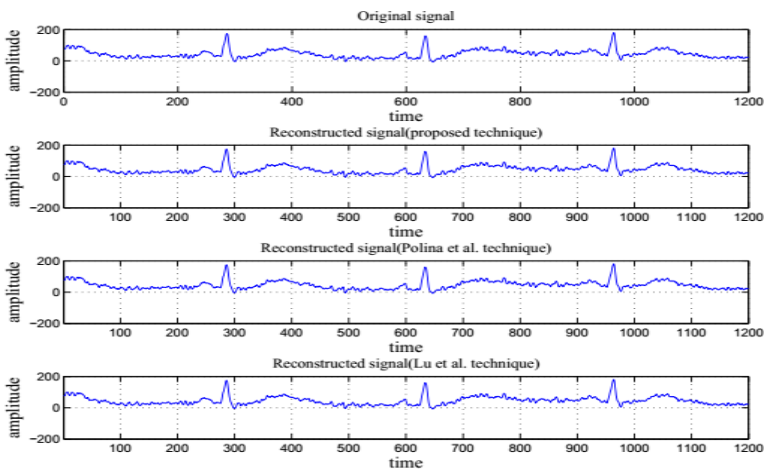

Fig. 5. Original signal and recovered signal for normal persons for record 100
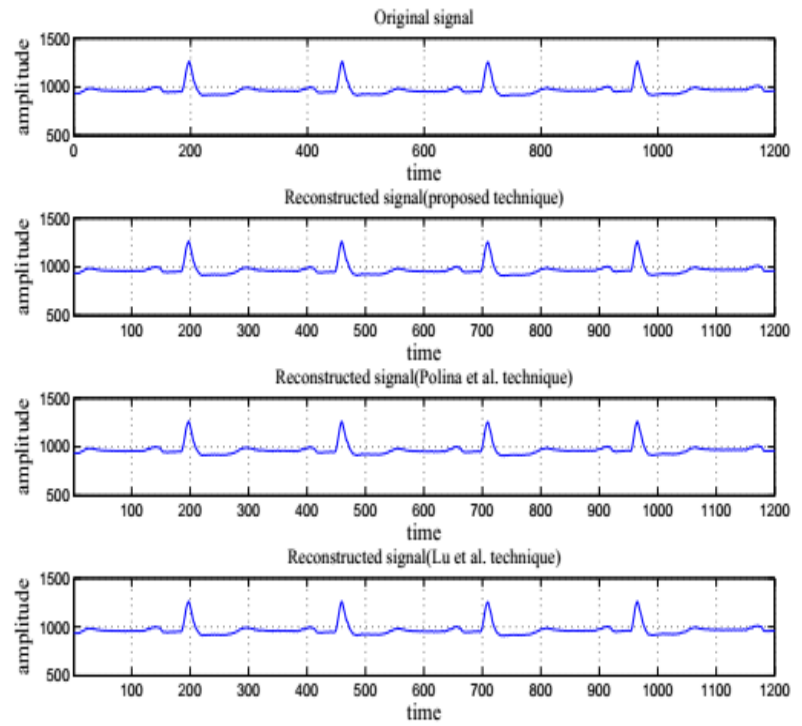

Fig.6. Original signal and recovered signal for normal persons for record 105

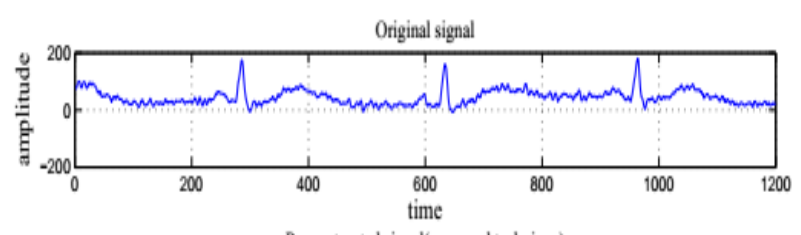

Reconstructed signal(proposed technique)

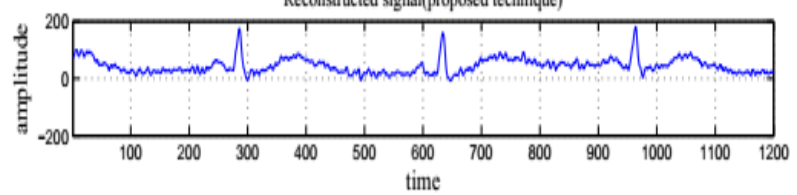

Reconstructed signal(Polina et al. technique)

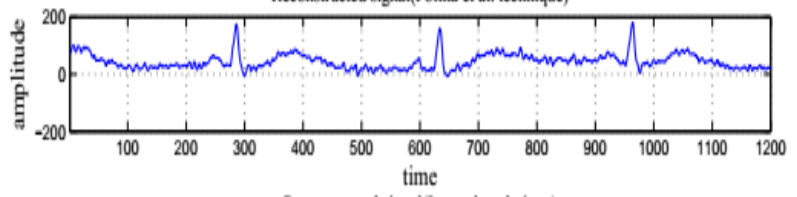

Reconstructed signal(Lu et al. technique)

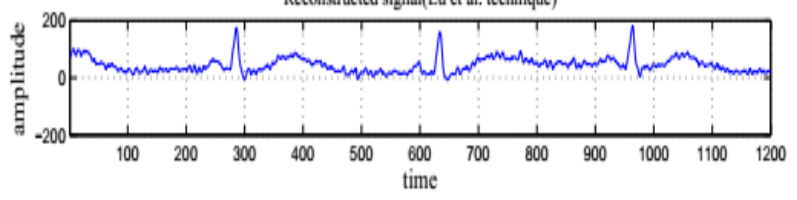

Fig.7. Original signal and recovered signal for patient persons for record 425

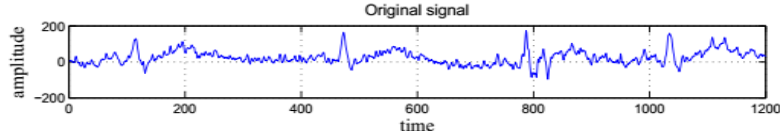

Reconstructed signal(proposed technique)

Reconstructed signal(Polina et al. technique)

Reconstructed signal(Lu et al. technique)

Fig.8. Original signal and recovered signal for patient persons for record 605

The proposed method performs preliminary reconstruction better than the previous approach for normal and patient person. Figures 5, 6, 7and 8 show this fact. These figures approve the superiority of the proposed algorithm. The study of the effects of increasing the number of samples on both CR and PRD for normal and patient persons is illustrated in the next figures. This study will prove the excellent performance of the proposed approach with respect to the two most recent techniques in this area of search. The results are taken for normal persons as well as for patient persons. In this way, the simulation results cover different cases to give more declaration of the priority of the proposed technique.
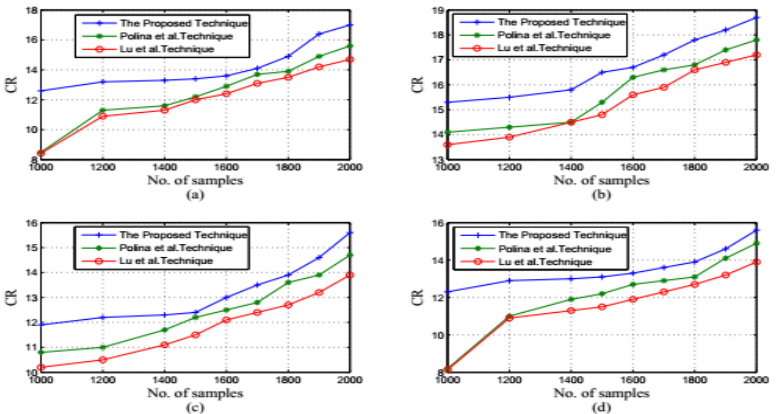

Fig. 9. The effect of increasing number of samples on CR for normal persons for Record 100 b) Record 105 c) Record 113 d) Record 119
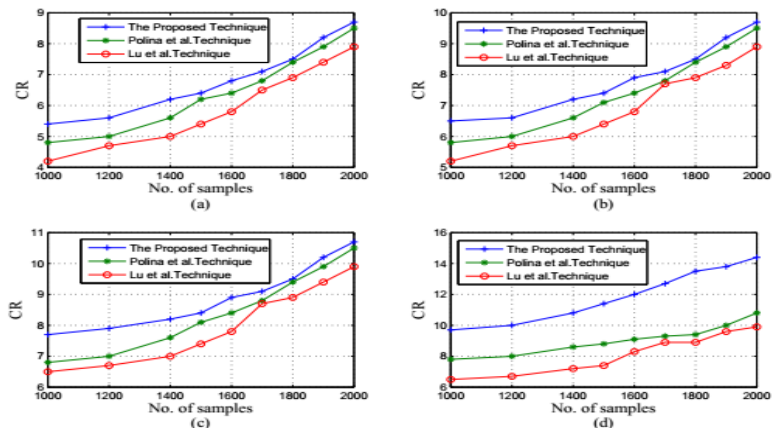

Fig.10. The effect of increasing number of samples on CR for patient persons for Record 420 b) Record 425 c) Record 605 d) Record 615

CR in CS method is considered to be the most important parameter to determine the robustness of any new technique that appears in this area of search. The researchers considered it as the challenge parameter that is directed toward the high 
performance. The simulation results indicate that $\mathrm{CR}$ in the proposed method achieved excellent results. This makes the proposed technique to be suggested in biomedical engineering. It makes also the researchers trend toward this technique as an excellent technique in this domain of search. Figures 8 and 9 demonstrate this fact. We can note from these figures also that an increasing in the number of samples leads to the increasing of CR.

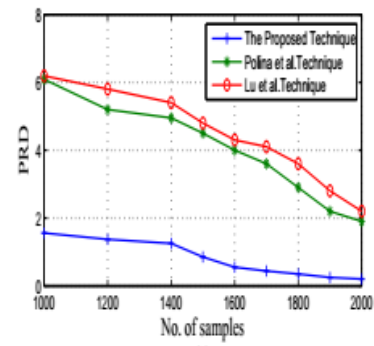

(a)

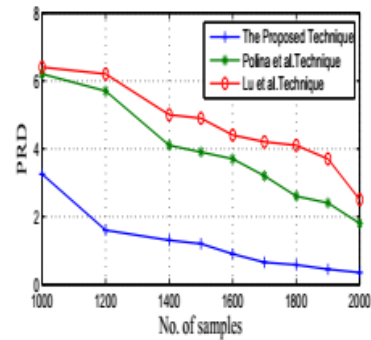

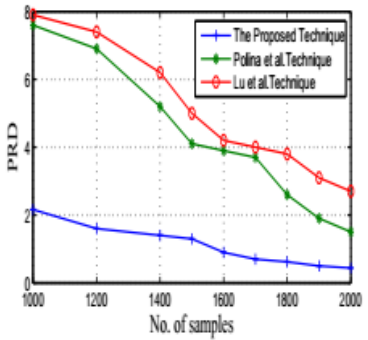

(b)

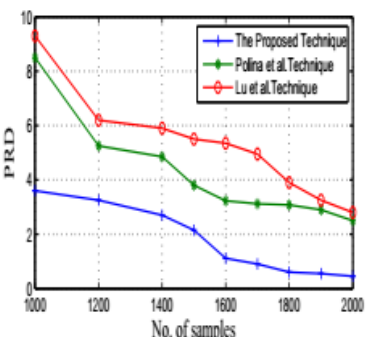

(d)
Fig. 11. The effect of increasing number of samples on PRD for normal persons for Record 100 b) Record 105 c) Record 113 d) Record 119

Figure 11 shows that the proposed technique has PRD with acceptable values. The values of PRD of the proposed technique for different number of samples are better than the two most recent techniques in the same domain of search. These two techniques used DWT to obtain sparse signal of ECG. But the proposed technique used ridgelet transform. In the next figure, the same simulation is done, but for the patient persons.

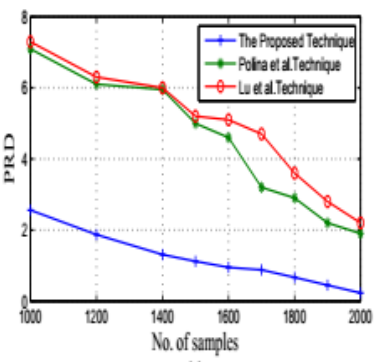

(a)

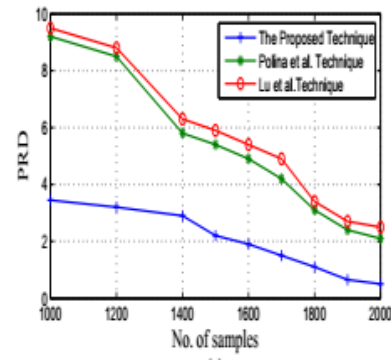

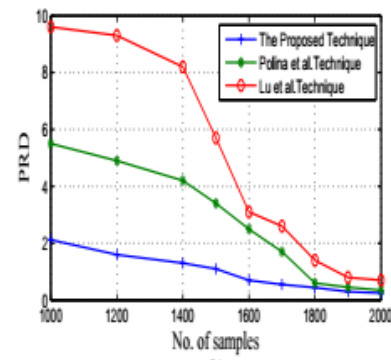

(b)

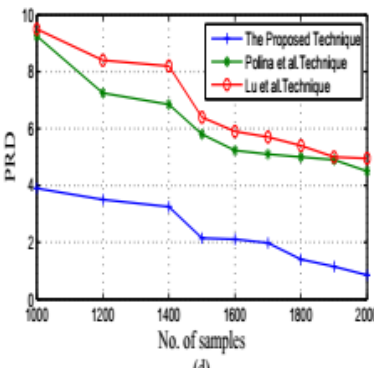

(d)
Fig. 12. The effect of increasing number of samples on PRD for patient persons for Record 420 b) Record 425 c) Record 605 d) Record 615

Figures 11 and 12 illustrate that to achieve minimum distortion between original signal and reconstructed signal, the proposed technique is suitable for that. This gives the proposed technique the robust in its domain of search.

Table 2. Performance comparison with previous compression algorithms for normal persons

\begin{tabular}{|c|c|c|c|c|c|}
\hline Record Number & Technique & CR & PRD & MSE & PSNR \\
\hline \multirow{3}{*}{100} & Proposed Technique & 12.6 & 1.56 & 0.079 & 14.57 \\
& Polina et al. Technique [20] & 8.49 & 6.09 & 0.09 & 13.5 \\
& Lu. et al. Technique [19] & 8.42 & 6.19 & 0.095 & 12.4 \\
\hline \multirow{3}{*}{105} & Proposed Technique & 15.2 & 2.16 & 0.08 & 19.2 \\
& Polina et al. Technique [20] & 14.1 & 7.6 & 0.087 & 15.9 \\
& Lu. et al. Technique [19] & 13.7 & 7.9 & 0.097 & 14.3 \\
\hline \multirow{3}{*}{113} & Proposed Technique & 12.9 & 3.25 & 0.09 & 19.7 \\
& Polina et al. Technique [20] & 10.9 & 6.2 & 0.092 & 17.6 \\
& Lu. et al. Technique [19] & 10.2 & 6.4 & 0.096 & 14.8 \\
\hline \multirow{3}{*}{119} & Proposed Technique & 12.2 & 3.6 & 0.088 & 22.3 \\
& Polina et al. Technique [20] & 8.15 & 8.5 & 0.095 & 15.4 \\
& Lu. et al. Technique [19] & 8.1 & 9.3 & 0.099 & 13.3 \\
\hline
\end{tabular}


Table 3. Performance comparison with previous compression algorithms for patient persons

\begin{tabular}{|c|c|c|c|c|c|}
\hline Record Number & Technique & CR & PRD & MSE & PSNR \\
\hline \multirow{3}{*}{420} & Proposed Technique & 5.4 & 2.56 & 0.087 & 16.57 \\
& Polina et al. Technique [20] & 4.8 & 7.09 & 0.098 & 15.5 \\
& Lu. et al. Technique [19] & 4.2 & 7.29 & 0.099 & 14.4 \\
\hline \multirow{3}{*}{425} & Proposed Technique & 6.5 & 2.12 & 0.091 & 18.7 \\
& Polina et al. Technique [20] & 5.8 & 5.5 & 0.094 & 17.5 \\
& Lu. et al. Technique [19] & 5.2 & 9.6 & 0.099 & 15.2 \\
\hline \multirow{3}{*}{605} & Proposed Technique & 7.7 & 3.45 & 0.0925 & 15.5 \\
& Polina et al. Technique [20] & 6.8 & 9.2 & 0.094 & 13.8 \\
& Lu. et al. Technique [19] & 6.5 & 9.5 & 0.098 & 12.3 \\
\hline \multirow{3}{*}{615} & Proposed Technique & 9.8 & 3.9 & 0.091 & 16.5 \\
& Polina et al. Technique [20] & 7.9 & 9.25 & 0.092 & 14.9 \\
& Lu. et al. Technique [19] & 6.2 & 9.5 & 0.097 & 13.8 \\
\hline
\end{tabular}

\section{FPGA REALIZATION}

The proposed design is simulated via FPGA to show how the proposed design achieved excellent results in terms of hardware implementation. The procedures done to clarify the algorithm of the proposed design can be summarized as follow. First, the matlab code was used to determine optimal parameters for wavelet transform. After that, the output of the matlab code was converted to fixed point representation. Next step, this fixed point can be used in the code of virtex -II pro.
The importance of these procedures is that the embedded PowerPC has no floating-point unit (FPU), and at the same time FPGAs don't support floating-point either. Although a FPU could be designed in an FPGA, they are resource intensive, and therefore can feasibly only support sequential operations. Rounding operation was performed on the signals before converting it into binary format. Figure 13 shows the timing diagram for the output signals using proposed design. Table 4 illustrates the resources used for the different designs compared with the proposed one.

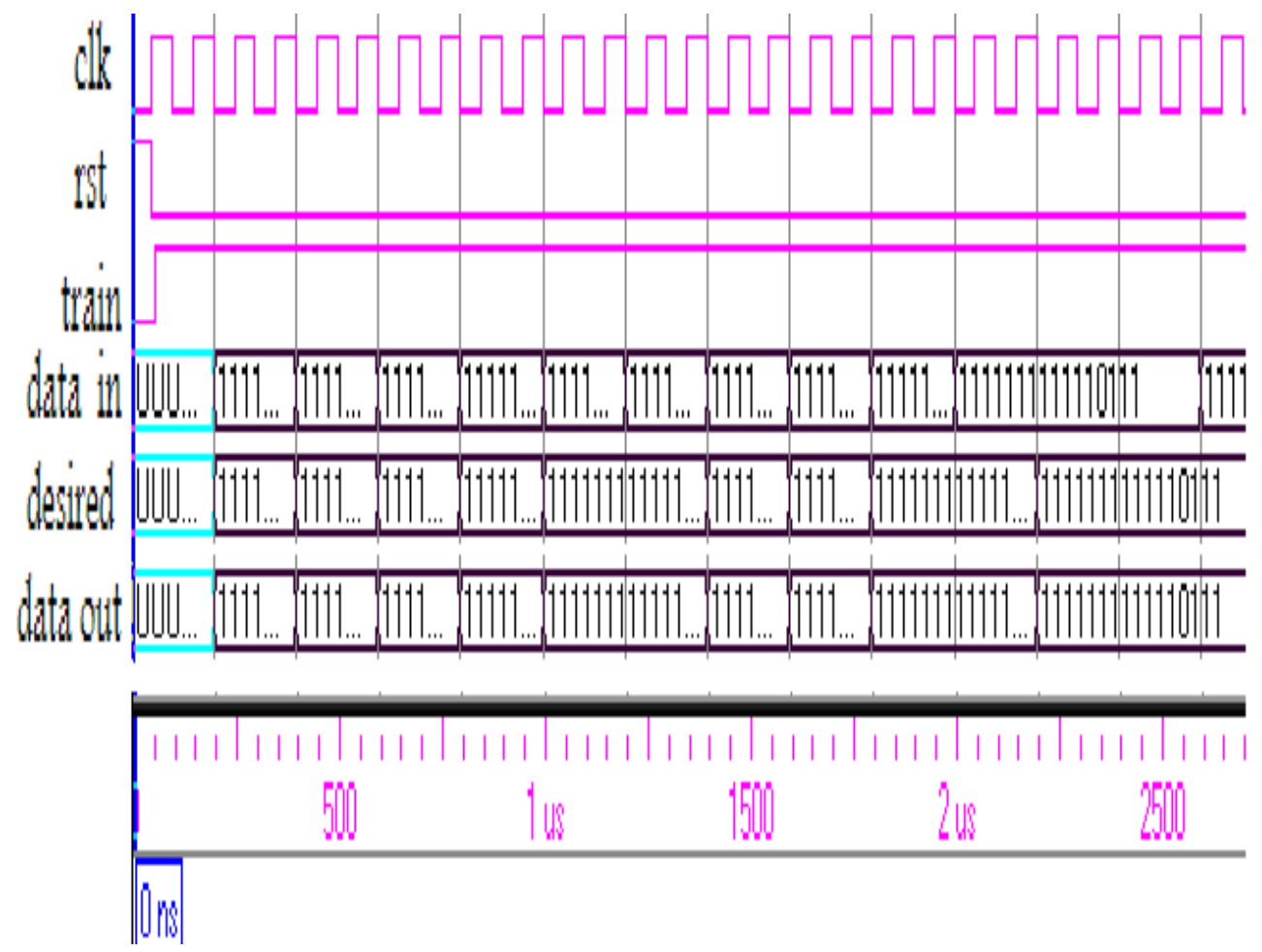

Fig. 13 Timing diagram for the output signals 
Table 4. Resources utilization of the proposed design compared with the previous works

\begin{tabular}{|c|c|c|c|c|}
\hline Logic Utilization & $\begin{array}{c}\text { Utilization Using FPGA } \\
\text { virtex-5[32] }\end{array}$ & $\begin{array}{c}\text { Utilization Using } \\
\text { FPGA virtex-6[33] }\end{array}$ & $\begin{array}{c}\text { Utilization Using } \\
\text { Kintex-7[34] }\end{array}$ & $\begin{array}{c}\text { Utilization Using The } \\
\text { proposed design }\end{array}$ \\
\hline Registers & $23.99 \%$ & $22.99 \%$ & $22.99 \%$ & $21.99 \%$ \\
\hline LUT & $65.22 \%$ & $64.18 \%$ & $63.99 \%$ & $63.56 \%$ \\
\hline Block RAM & $43.28 \%$ & $41.58 \%$ & $40.28 \%$ & $37.28 \%$ \\
\hline DSP48Es & $54.83 \%$ & $52.81 \%$ & $50.17 \%$ & $46.83 \%$ \\
\hline
\end{tabular}

\section{CONCLUSIONS}

This paper proposed a complete system-level comparison for enhancement of sparse signal of ECG. This comparison is between a new CS-based on obtaining sparse signal of ECG by using ridgelet transform and the state-of-the-art DWT that is based on embedded ECG compression algorithms. Compressive Sensing is a useful tool for eliminating the inefficiencies caused by traditional signal processing algorithms. Compressive Sensing is an effective method to make data compressed for ECG signals with high compression ratio and good quality of reconstruction. Experimental results show that the ridgelet transform compression method has better performance based on Compression Ratio (CR), Percentage Root Square Difference (PRD), Peak Signal to Noise Ratio (PSNR) and Mean Square Error (MSE).It is clear that the proposed algorithm has the highest CR and also an acceptable PRD. CS derived compression coefficients can use a set of connection weights extracted from a trained Spiking Neural Network. The proposed design uses parallel Virtex field programmable gate array devices (FPGA). Curvelet is better area optimization. It achieves a great result in increasing the throughput. This architecture uses the clock frequency with high speed. In this proposed design, the area utilization is reduced below the previous approaches. The used resources in the proposed approach show the efficient of this algorithm for FPGA realization. This approach can certainly be the domain of future studies.

\section{REFERENCES}

[1] Mohammed M. Abo-Zahhad, Aziza. I. Hussein and Abdelfatah M. Mohamed, "Compressive Sensing Algorithms for Signal Processing Applications: A Survey", Int. J. Communications, Network and System Sciences 2015, Vol. 8, PP. 197-216.

[2] Yong Wang, Zhoushi Yang and Yiran Shen, "CS Collector: A New Approach for Data Collection in Wireless Sensor Networks Based on Two-Dimensional Compressive Sensing", Sensors 2016, Vol. 16, PP. 1-16.

[3] Matthew Hawes, Wei Liu and Lyudmila Mihaylova, "Compressive Sensing Based Design of Sparse Tripole Arrays", Sensors 2015, Vol. 15, PP. 31056-31068.

[4] Vertterli M., Marziliano P. and Blu T. S., "Sampling signals with finite rate of innovation", IEEE Transactions on Signal Processing 2002, Vol. 50, PP. 352-358.

[5] J. Cardenas-Barrera, J. Lorenzo-Ginori and E. Rodriguez-Valdivia, "A wavelet-packets based algorithm for EEG signal compression", Med. Informatic. and Internet in Med 2004, Vol. 29, PP. 15-27.

[6] Mohammed M. Abo-Zahhad, Aziza I. Hussein and Abdelfatah M. Mohamed, "Compression of ECG Signal Based on Compressive Sensing and the Extraction of
Significant Features", Int. J. Communications, Network and System Sciences 2015, Vol. 8, PP. 97-117.

[7] Hossein Mamaghanian, Nadia Khaled, David Atienza and Pierre Vandergheynst, "Compressed Sensing for Real-Time Energy-Efficient ECG Compression on Wireless Body Sensor Nodes", IEEE tansactions on biomedical engineering 2011, Vol. 58, PP. 2456-2466.

[8] Hend Fathey, Eman Mohamed, Ashraf Mohamed and Wagdy Anis, "Enhancement of ECG Signal", International Journal of Computer Applications 2016, Vol.145, PP.12-16.

[9] Brikena Xhaja, Eglantina Kalluci and Ligor Nikolla, "Wavelet Transform Applied in ECG Signal Processing", European Scientific Journal 2015, Vol. 11, PP. 305-312.

[10] Chen F., Chandrakasan and Stojanovic, "Design and Analysis of a Hardware-Efficient Compressed Sensing Architecture for Data Compression in Wireless Sensors", IEEE Journal of Solid-State Circuits 2012, Vol. 47, PP. 744-756.

[11] Namrata and Vaswani, "LS-CS-Residual (LS-CS): Compressive Sensing on Least Squares Residual", IEEE Transactions on Signal Processing 2010, Vol. 58, PP. 944-958.

[12] S. Edward Jeroa, Palaniappan Ramua and S.Ramakrishnanb, "ECG Steganography Using Curvelet Transform", Biomedical Signal Processing and Control 2015, 35, 519-530.

[13] E. J. Cand'es, Ph.D. thesis, Department of Statistics, Stanford University, "In Ridgelets: Theory and Applications", Editor, F., Meditor, A., Eds.; Publishing House: City, Country, 1998; PP. 32-58.

[14] E. J. Cand'es and D. L. Donoho, Ridgelets: "A key to higherdimensional intermittency? Phil. Trans. R. Soc. Lond. A.", 1999, Vol. 10, PP. 2495-2509.

[15] S. R. Deans, "The Radon Transform and Some of Its Applications", John Wiley and Sons 1983, Vol. 10, PP. 154-169.

[16] Vijay, Gowri, Velmurugan and Dr.AM.Basha, "Detection and Extraction of $\mathrm{P}$ Wave and $\mathrm{T}$ Wave in ECG to Improve Sensitivity for E-Health Monitoring", International Journal of Communication and Computer Technologies 2016, Vol. 4, PP. 4020-4024.

[17] Mohammed Abo-Zahhad, Sabah M.Ahmed and Ahmed Zakaria, "An efficient Technique for Compressing ECG Signals Using QRS Detection, Estimation and 2D-DWT Coefficients Thresholding", Modeling and Simulation in Engineering 2012, Vol. 20, PP. 1-10. 
[18] Y. Eldar and M. Mishali, "Robust recovery of signals from a structured union of subspaces", IEEE Transactions Info. Theory, 2009, Vol. 55, PP. 53025316.

[19] Lu., Z., Kim and Pearlman, "Wavelet Compression of ECG Signals by the Set Partitioning in Hierarchical Trees Algorithm", IEEE Transactions on Biomedical Engineering 2000, Vol. 47, PP. 849-856.

[20] Luisa F. Polania, Rafael E. Carrillo, Manuel BlancoVelasco and Kenneth E. Barner, "Compressed Sensing Based Method for ECG Compression", IEEE International Conference on Acoustics, Speech and Signal Processing (ICASSP) 2011, PP. 761-764.

[21] Bharti Salsekar, "Filtering of ECG Signal Using Butterworth Filter and its Feature Extraction", International Journal of Engineering Science and Technology 2018, Vol. 4, PP. 1292-1298.

[22] Surekha K.S. and B.P. Patil, "ECG Signal Compression Using the High Frequency Components of Wavelet Transform", International Journal of Advanced Computer Science and Applications 2016, Vol. 7, PP. 311-315.

[23] Mile Petkovski, Sofija Bogdanova, Momcilo Bogdanov, "A Simple Adaptive Sampling Algorithm", $14^{\text {th }}$ Telecommunications forum TELFOR 2006, PP. 329-332.

[24] Skander Bensegueni and Abdelhak Bennia, "ECG Signal Compression Using a Sinusoidal Transformation of Principal Components", International Journal of Software engineering and its applications 2017, Vol. 10, PP. 59-68.

[25] Surekha K.S. and B. P. Patil, "Transform Based Techniques for ECG Signal Compression", International Journal of Signal Processing, Image Processing and Pattern Recognition 2016, Vol. 11, PP. 6139-6143.

[26] P. S. Hiremath, S. Shivashankar and Jagadeesh Pujari, "Wavelet Based Features for Color Texture Classification with Application to CBIR", International Journal of Computer Science and Network Security (IJCSNS) 2006, Vol. 6, PP. 101-106.
[27] S. Arulselvi and P. Mangaiyarkarasi, "A New Digital Image Watermarking Based on Finite Ridgelet Transform and Extraction Using ICA", DETECT IEEE 2011, Vol. 34, PP. 837-841.

[28] M. Y. Abbass, S. El-Rabaie and F. E. Abd El-Samie, "Efficient Blind Image Separation Using Finite Ridgelet Transform", ICCTA, 2013, PP. 29-31.

[29] Karishma Qureshi and V. P. Patel, "Efficent Data Compression of ECG Signal Using Discrete Wavelet Transform", International Journal of Research in Engineering and Technology, 2013, Vol. 2, PP. 696-699.

[30] Desai Siddhi and Nakrani Naitik, "Improved Performance of Compressive Sensing for Speech Signal with Orthognal Symmetric Toeplitz Matrix", International Journal of Signal Processing, Image Processing and Pattern Recognition, 2017, Vol. 7, PP. 371-380.

[31] Akanksha Mishra, Falgun Thakkar, Chintan Modi and Rahul Kherl, "Comparative Analysis of Wavelet Basis Functions for ECG Signal Compression through Compressive Sensing", International Journal of Computer Science and Telecommunications, 2012, Vol. 3, PP. 23-51.

[32] L. Bai, P. Maechler, M. Muehlberghuber, and H. Kaeslin, "High speed compressed sensing reconstruction on FPGA using OMP and AMP", in Proceedings of the 19th IEEE International Conference on Electronics, Circuits, and Systems (ICECS '12), IEEE, Seville, Spain, December 2012, pp. 53-56.

[33] J. L. V. M. Stanislaus and T. Mohsenin, "Lowcomplexity FPGA implementation of compressive sensing reconstruction", in Proceedings of the International Conference on Computing, Networking and Communications (ICNC '13), January 2013 pp. 671-675.

[34] F.B.Ren, R.Dorrace, W.Y.Xu, and D.Markovi'c, "A single-precision compressive sensing signal reconstruction engine on FPGAs", in Proceedings of the 23rd International Conference on Field Programmable Logic and Applications (FPL '13), IEEE, Porto, Portugal, September 2013, pp.1-4. 